\title{
Estimation of Sideslip Angle Based on Extended Kalman Filter
}

\author{
Yupeng Huang, ${ }^{1}$ Chunjiang Bao, ${ }^{1}$ Jian $\mathrm{Wu}^{1,2}$ and Yan $_{\mathrm{Ma}}{ }^{1}$ \\ ${ }^{1}$ School of Mechanical and Automotive Engineering, Liaocheng University, Liaocheng, Shandong 252000, China \\ ${ }^{2}$ Department of Automotive Engineering, Tsinghua University, Haidian District, Beijing 100084, China \\ Correspondence should be addressed to Chunjiang Bao; baochunjiang@lcu.edu.cn
}

Received 14 October 2016; Accepted 5 March 2017; Published 28 March 2017

Academic Editor: Jit S. Mandeep

Copyright (C) 2017 Yupeng Huang et al. This is an open access article distributed under the Creative Commons Attribution License, which permits unrestricted use, distribution, and reproduction in any medium, provided the original work is properly cited.

\begin{abstract}
The sideslip angle plays an extremely important role in vehicle stability control, but the sideslip angle in production car cannot be obtained from sensor directly in consideration of the cost of the sensor; it is essential to estimate the sideslip angle indirectly by means of other vehicle motion parameters; therefore, an estimation algorithm with real-time performance and accuracy is critical. Traditional estimation method based on Kalman filter algorithm is correct in vehicle linear control area; however, on low adhesion road, vehicles have obvious nonlinear characteristics. In this paper, extended Kalman filtering algorithm had been put forward in consideration of the nonlinear characteristic of the tire and was verified by the Carsim and Simulink joint simulation, such as the simulation on the wet cement road and the ice and snow road with double lane change. To test and verify the effect of extended Kalman filtering estimation algorithm, the real vehicle test was carried out on the limit test field. The experimental results show that the accuracy of vehicle sideslip angle acquired by extended Kalman filtering algorithm is obviously higher than that acquired by Kalman filtering in the area of the nonlinearity.
\end{abstract}

\section{Introduction}

In the recent years, with the improvement of the road conditions and the progress of automobile technology, the speed of the car is also accelerating; however, the proportion of traffic accidents caused by the instability of vehicle is also increasing [1]. Therefore, it is an important subject for modern automobile to improve the safety. In terms of vehicle stability control system, the vehicle sideslip angle is a very important control variable [2].

In the low adhesive road, the vehicle is easy to sideslip because of the tire lateral force's saturation; although the yaw rate remains in a small range, the vehicle has lost the balance [3]. In other words, the actual yaw rate is the same as the ideal value, the sideslip angle will increase quickly and exceed critical value, and then the vehicle loses the balance. Therefore, in the low adhesive road, the sideslip angle can be better describing the real situation of the vehicle $[4,5]$. The yaw rate can be obtained directly by the yaw rate sensor and because of the cost there is no sensor to directly measure the sideslip angle. The sideslip angle can only be estimated by measuring the motion state parameters of other vehicles and using the estimation method [6]. There are many methods to estimate sideslip angle such as the original integration method, the simple Kalman filter to the present neural network, and the fuzzy logic method. There are great improvements in estimation accuracy, computational efficiency, and robustness of the algorithm [7]. The advantages of the direct integration method are simplicity, being easy to implement, small amount of calculation, and good realtime performance. The disadvantage of the direct integration method is that, with the increase of time, the accumulation of the error increases, so that the estimated value is greatly deviated from the true value $[8,9]$. To estimate the vehicle sideslip angle based on fuzzy logic and vehicle kinematics model combining test, the results show that the robustness and accuracy of the method are good, and the response frequency is high. Then, it could meet the control needs of ESP $[10,11]$. In the above algorithm, the author takes the real-time, robustness, accuracy and other reasons into consideration, based on the application of Kalman filtering algorithm in the actual project [12]. However, with the limitation of the linear tire model itself, the filter algorithm of Kalman estimation method is accurate within vehicles 


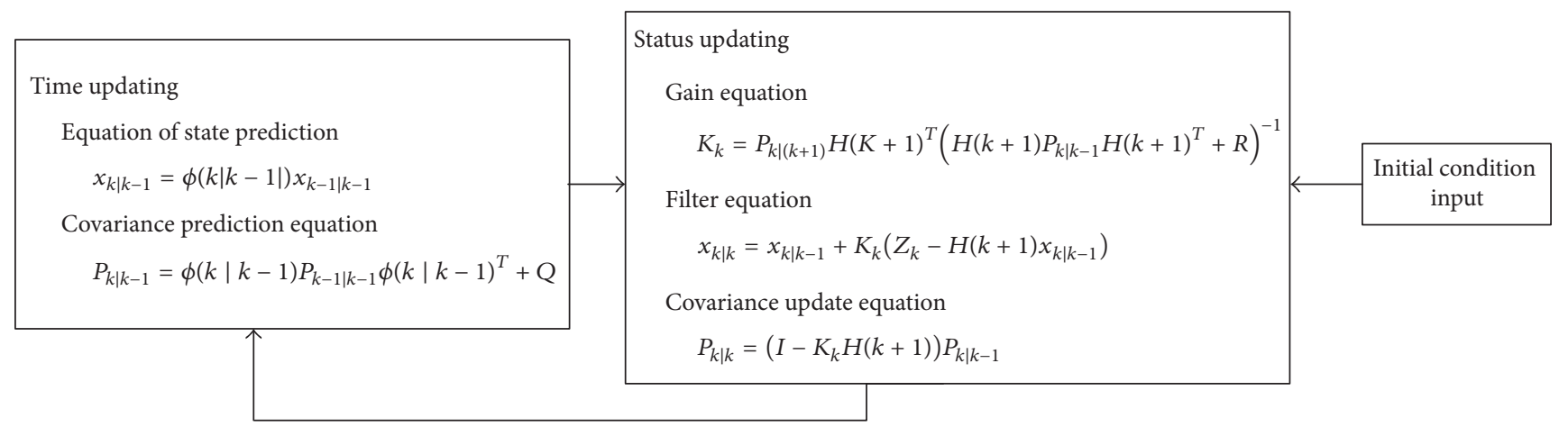

FIGURE 1: EKF algorithm flowchart.

linear control area and it is not enough for vehicle stability control system which can be triggered only under extreme conditions [3]. For example, the nonlinear characteristics of the vehicle are more obvious on the low adhesive road, but the extended Kalman filtering method can obtain good effects even when vehicle dynamics are in nonlinear area because the extended Kalman filtering method considers the nonlinear characteristics of tires [13]. Therefore, this paper uses the extended Kalman filtering method to study the sideslip angle of the vehicle.

\section{Extended Kalman Filtering Theory}

2.1. Kalman Filtering Theory. Kalman filtering method is a linear system estimation method. The process can be divided into two parts [14]:

(i) Time updating:

$$
\begin{aligned}
& x_{k \mid k-1}=A x_{k-1 \mid k-1}+B u_{k-1} \\
& P_{k \mid k-1}=A P_{k-1 \mid k-1} A^{T}+Q .
\end{aligned}
$$

(ii) Measurement updating:

$$
\begin{aligned}
K_{k} & =P_{k \mid k-1} H^{T}\left(H P_{k \mid k-1} H^{T}+R\right)^{-1} \\
x_{k \mid k} & =x_{k \mid k-1}+K_{k}\left(y_{k}-H x_{k \mid k-1}\right) \\
P_{k \mid k} & =\left(I-K_{k} H\right) P_{k \mid k-1} .
\end{aligned}
$$

In this formula, $x_{k}$ is the state vector for $k$ moment in the system, $u_{k}$ is the system input, $y_{k}$ is the observation vector, and $Q, R$ is the covariance matrix for process noise and measurement noise.

2.2. Extended Kalman Filtering Theory. Kalman filtering algorithm has low precision for some systems with strong nonlinearity. It generally uses extended Kalman filter (EKF) for the state estimation of nonlinear systems such as automobiles. EKF transfers nonlinear model into linear model, so that Kalman filter can be used for nonlinear systems.

The processes of EKF are as shown in Figure 1.
Usually, the state space equation and the observation equation can be expressed by the following formula:

$$
\begin{aligned}
& x_{k}=f\left(x_{k-1}, u_{k-1}\right) \\
& y_{k}=h\left(x_{k}\right) .
\end{aligned}
$$

In the nonlinear state equation and observation equation ((3) and (4)), extended Kalman filtering algorithm can be expanded through Taylor series. The specific approach of linear approximation of nonlinear equations (3) is to make linearization by state equation and measurement equation for nonlinear system (i.e., the Jacobian matrix) and then estimate it. The specific steps of the EKF method are as follows.

The state update process of EKF is as follows:

$$
\begin{aligned}
& x_{k \mid k-1}=f\left(x_{k-1 \mid k-1}, u_{k-1}\right) \\
& P_{k \mid k-1}=A_{k} P_{k-1 \mid k-1} A_{k}^{T} .
\end{aligned}
$$

The measurement update process of EKF is as follows:

$$
\begin{aligned}
K_{k} & =P_{k \mid k-1} H_{k}^{T}\left(H P_{k \mid k-1} H_{k}^{T}+V_{k} R_{k} V_{k}^{T}\right)^{-1} \\
x_{k \mid k} & =x_{k \mid k-1}+K_{k}\left(y_{k}-h\left(x_{k \mid k-1}\right)\right) \\
P_{k \mid k} & =\left(I-K_{k} H_{k}\right) P_{k \mid k-1} .
\end{aligned}
$$

In the formula, $A$ is the Jacobian matrix of $f$ to $x$ :

$$
A(k \mid k-1)_{[i, j]}=\frac{\partial f_{[i]}}{\partial x_{[j]}}\left(x_{k-1 \mid k-1}, u_{k-1}\right) \text {. }
$$

In the formula, $H$ is the Jacobian matrix of $f$ to $x$ :

$$
H(k)_{[i, j]}=\frac{\partial h_{[i]}}{\partial x_{[j]}}\left(x_{k \mid k-1}\right) .
$$

\section{Simulation Analysis of Sideslip Angle Estimation Based on Extended Kalman Filtering Algorithm}

The extended Kalman filtering estimation algorithm is based on the 2-degree-of-freedom vehicle dynamics model (shown in Figure 2), and the tire model is based on MF formula. 


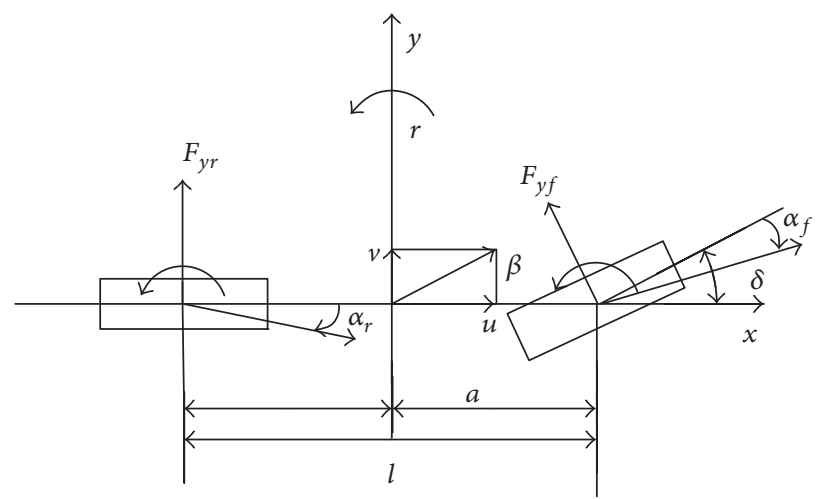

FIGURE 2: 2-dof vehicle dynamic model.

3.1. Sideslip Angle Estimation Based on Extended Kalman Filtering. According to the 2-degree-of-freedom vehicle model

$$
\begin{aligned}
m u(\dot{\beta}+r) & =F_{y f}+F_{y r} \\
I_{z} \dot{r} & =F_{y f} \cdot a+F_{y r} \cdot b \\
\alpha_{f} & =-\left(\delta-\operatorname{arctg}\left(\beta+\frac{a r}{u}\right)\right) \\
\alpha_{r} & =\operatorname{arctg}\left(\beta-\frac{b r}{u}\right)
\end{aligned}
$$

and MF tire

$$
\begin{aligned}
F_{y f} & =D_{f} \sin \left[C_{f}\right. \\
\cdot & \left.\arctan \left\{B_{f} \alpha_{f}-E_{f}\left(B_{f} \alpha_{f}-\arctan \left(B_{f} \alpha_{f}\right)\right)\right\}\right] \\
F_{y r} & =D_{r} \sin \left[C_{r}\right. \\
& \left.\cdot \arctan \left\{B_{r} \alpha_{r}-E_{r}\left(B_{r} \alpha_{r}-\arctan \left(B_{r} \alpha_{r}\right)\right)\right\}\right],
\end{aligned}
$$

establish the following formula:

(1) Establish the nonlinear state equation as

$$
\begin{gathered}
\dot{\beta}=\frac{F_{y f}+F_{y r}}{m u}-r \\
\dot{r}=\frac{a F_{y f}-b F_{y r}}{I_{z}}
\end{gathered}
$$

Measurement equation:

$$
\begin{aligned}
& a_{y}=\frac{F_{y f}+F_{y r}}{m}-r \\
& r=r
\end{aligned}
$$

Put $F_{y f}, F_{y r}$ with MF tire formula, state quantity is $x=$ $\left[\begin{array}{ll}\beta & r\end{array}\right]^{T}$, measurement is $y=\left[\begin{array}{ll}a_{y} & r\end{array}\right]^{T}$, and control input is $u=\delta$.
TABLE 1: Parameters and meaning.

\begin{tabular}{lc}
\hline Parameter & Meaning \\
\hline$a$ & The vehicle centroid to the front axle \\
$u$ & Vehicle quality \\
$\beta$ & The longitudinal velocity \\
$r$ & Sideslip angle \\
$F_{x}$ & Yaw rate \\
$F_{z}$ & Tire longitudinal force \\
$b$ & Vertical force of tire \\
$I_{z}$ & The vehicle centroid to the rear axle \\
$\alpha_{f}$ & Moment of inertia of vehicle around the lead \\
$\alpha_{r}$ & vertical shaft \\
$\delta$ & The front wheel sideslip angle \\
$F_{y}$ & Thear wheel sideslip angle \\
$l$ & Tire lateral force \\
\hline
\end{tabular}

(2) The linear model (take Jacobian matrix for state equation and measurement equation) is as follows:

$$
\begin{gathered}
A(t)=\left[\begin{array}{ll}
\frac{\partial f_{1}}{\partial \beta} & \frac{\partial f_{1}}{\partial \gamma} \\
\frac{\partial f_{2}}{\partial \beta} & \frac{\partial f_{2}}{\partial \gamma}
\end{array}\right] \\
H(t)=\left[\begin{array}{ll}
\frac{\partial h_{1}}{\partial \beta} & \frac{\partial h_{1}}{\partial \gamma} \\
\frac{\partial h_{2}}{\partial \beta} & \frac{\partial h_{2}}{\partial \gamma}
\end{array}\right] .
\end{gathered}
$$

Put the discretization of the equation of state " $A(k \mid k-$ 1) $=I+A(t) * T$ " and the discretization of the measurement equation " $H(k)=H(t)$ " in the five formulas of EKF status update and measurement update. Table 1 indicates various parameters and variables of automobile. 
Main interface

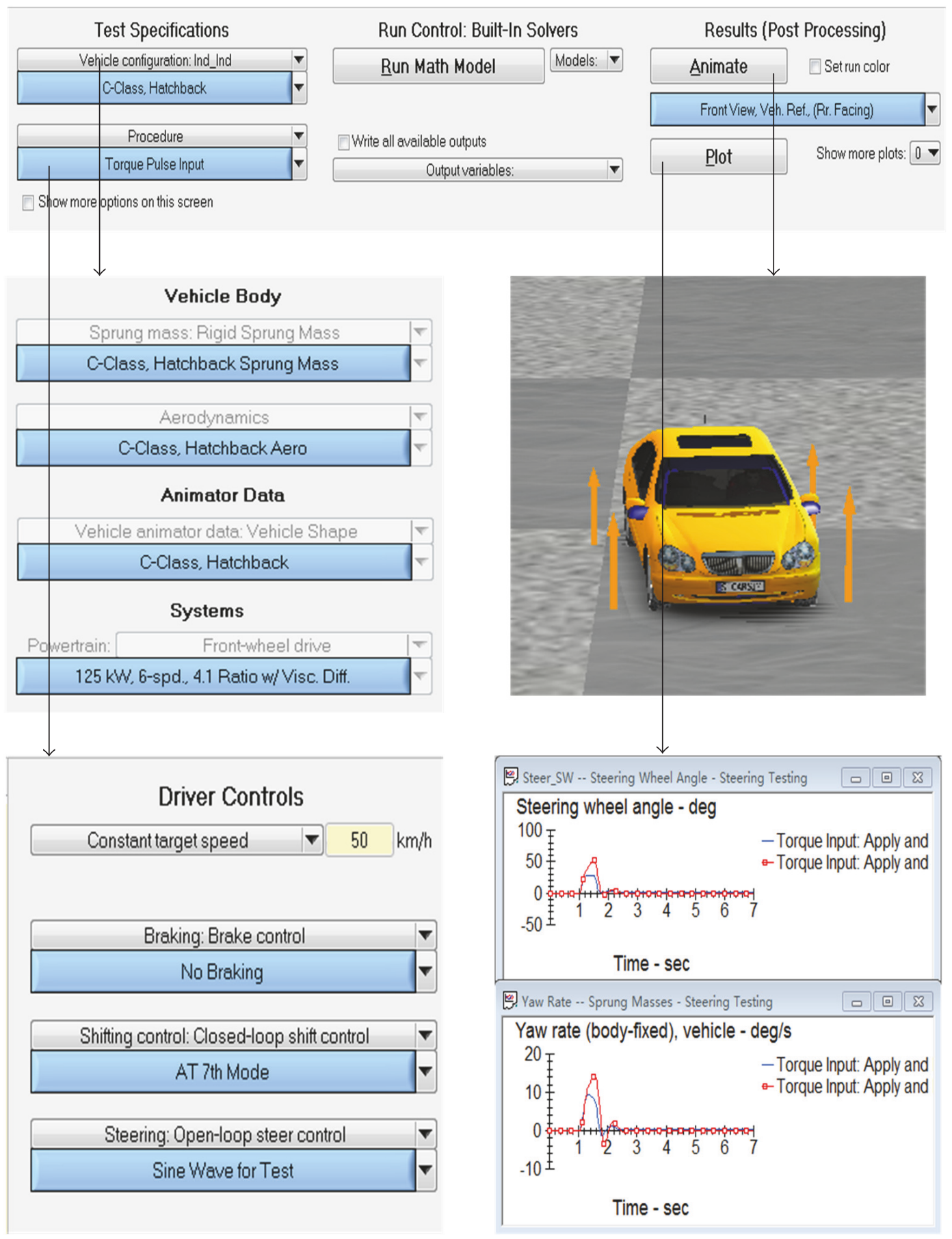

FIgURE 3: The main interface and constitution of Carsim.

3.2. Sideslip Angle Estimation Based on EKF Simulation. In order to verify the effect of extended Kalman filtering algorithm sideslip angle estimation, we carried out simulation verification to a car. The simulation adopts a typical double lane condition, in order to verify the accuracy of the extended Kalman filtering sideslip angle estimation and make the vehicle lateral acceleration as fast as possible, so as to make the vehicle into the nonlinear control area.

3.2.1. Joint Simulation of Carsim-Simulink. The main interface of Carsim includes four parts: vehicle characteristics interface, test environment interface, simulation solver 


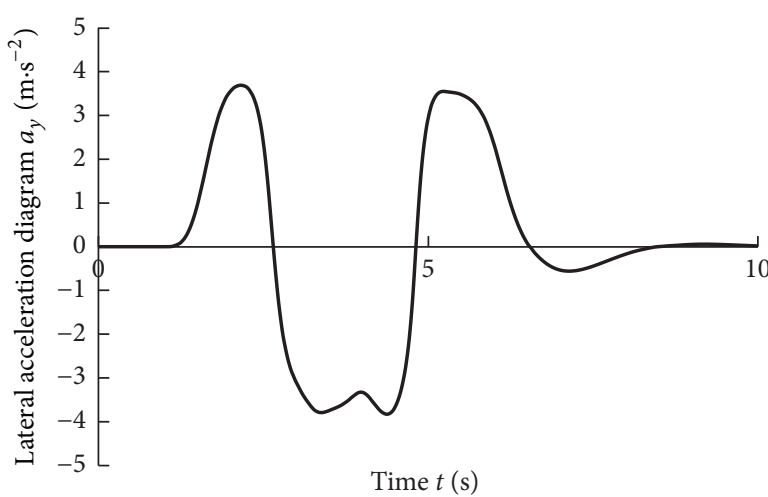

(a) Lateral acceleration diagram

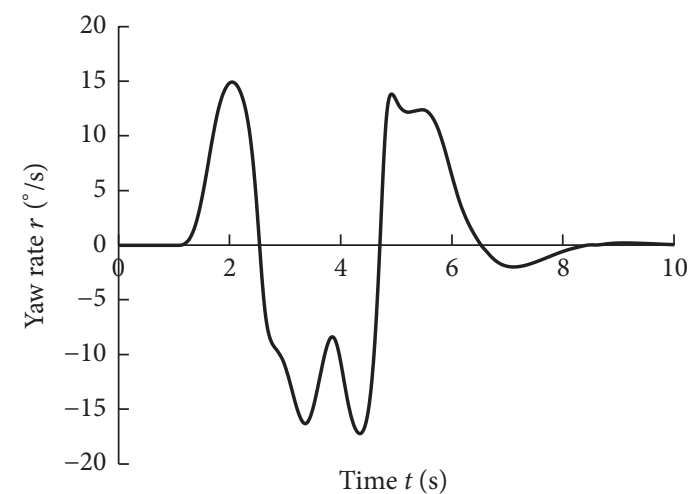

(b) Yaw rate

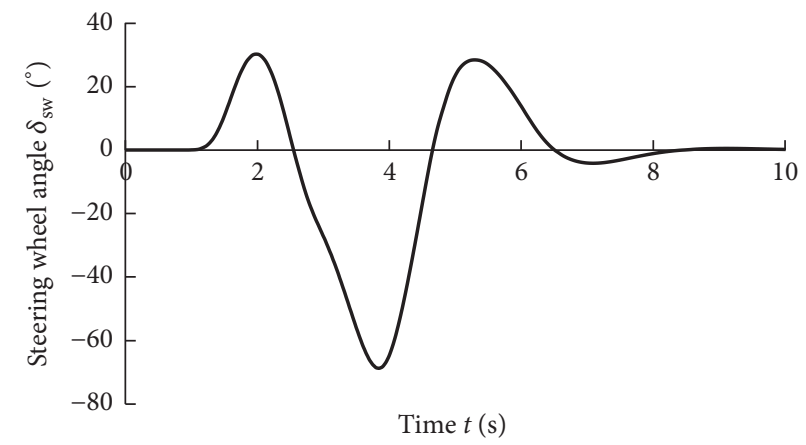

(c) Steering wheel angle

FIGURE 4: Vehicle status $(\mu=0.45)$.

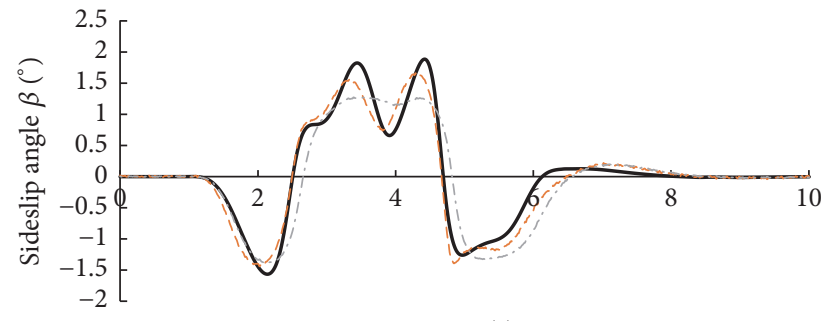

Time $t(\mathrm{~s})$

$$
\begin{array}{ll}
\text { - } & \text { Beta_real } \\
\ldots \text {-. } & \text { Beta_EKF } \\
\ldots & \text { Beta_KF }
\end{array}
$$

FIGURE 5: The contrast of sideslip angle simulation value and estimated value $(\mu=0.45)$.

interface, and 3D animation and experimental curve drawing interface. The relationship between the four parts and the main interface of Carsim is shown in Figure 3. Table 2 lists the parameters of vehicle settings in Carsim.

3.2.2. Wet Cement Pavement. Take the double lane change simulation of $95 \mathrm{~km} / \mathrm{h}$ on the simulation of ground with adhesion coefficient of 0.45 and use the extended Kalman filtering technology to recognize the sideslip angle. The result is shown in Figures 4 and 5.
Note from Figures 4(b) and 4(c) that the relationship between the yaw rate and the steering wheel angle is not linear, which indicates that the vehicle dynamics have entered the nonlinear region.

Note from Figure 5 that when we take the $4 \mathrm{~s}$, there are largely different Kalman filtering estimation and simulation of the actual value. Overall, the accuracy of the sideslip angle estimation is higher than the accuracy of Kalman filtering algorithm.

3.2.3. Ice and Snow Road. When the vehicle enters strongly nonlinear region, in order to verify the effect of the extended Kalman filtering algorithm to estimate the sideslip angle, then take the sideslip angle estimation simulation on low attachment snow ground (the ground adhesion coefficient is 0.2 ).

Take the double lane change simulation of $55 \mathrm{~km} / \mathrm{h}$ on the snow ground with round adhesion coefficient of 0.2 and then use the extended Kalman filtering algorithm to identify the sideslip angle. The result is shown in Figures 6 and 7.

In the snow and other low adhesion roads, the vehicle easily enters the nonlinear region. From Figure 7, we can see that when the vehicle is in its limit operation, the difference between the sideslip angle curve Kalman filtering algorithm estimated and actual sideslip angle is large and there is also a difference between the extended Kalman filtering estimation and simulation of the actual value, but the curve is relatively close; overall, the accuracy of the sideslip angle estimation is higher than the accuracy of Kalman filtering algorithm. 
Table 2: Parameters of vehicle settings in Carsim.

\begin{tabular}{lcc}
\hline Variable symbol & Variable name (unit) & Variable value \\
\hline$m$ & Vehicle quality $(\mathrm{kg})$ & 1247 \\
$a$ & The vehicle centroid to the front axle $(\mathrm{m})$ & 1.016 \\
$b$ & The vehicle centroid to the rear axle $(\mathrm{m})$ & 1.562 \\
$I_{z}$ & Moment of inertia about the $z$-axis $\left(\mathrm{kgm}^{\wedge} 2\right)$ & 1523 \\
\hline
\end{tabular}

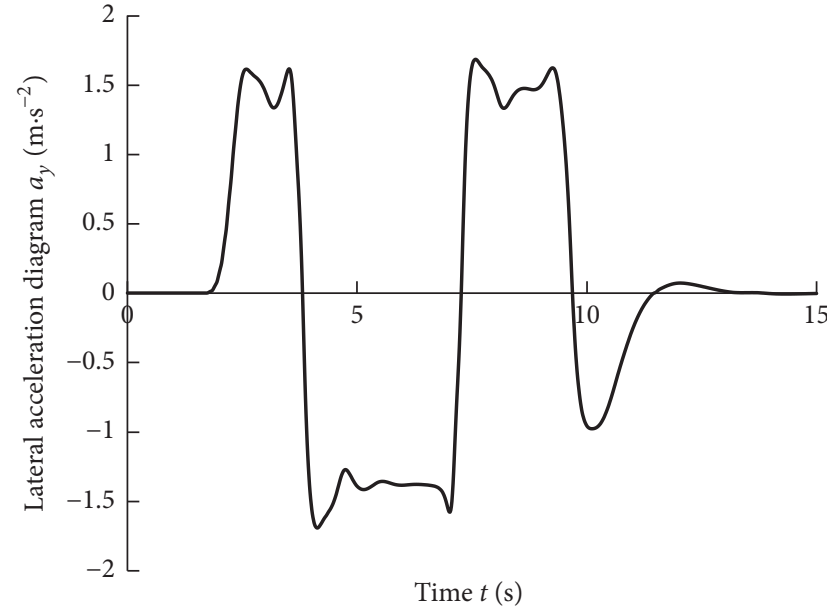

(a) Lateral acceleration diagram

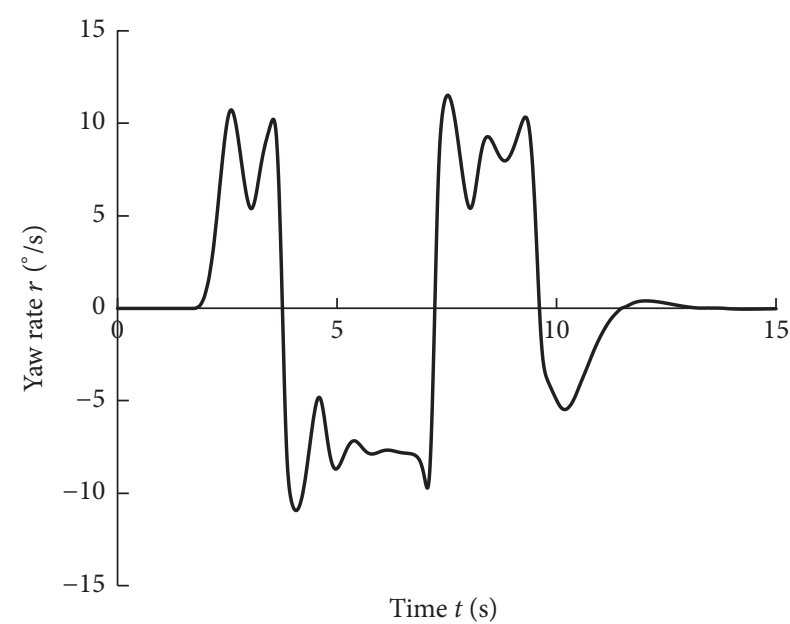

(b) Yaw rate

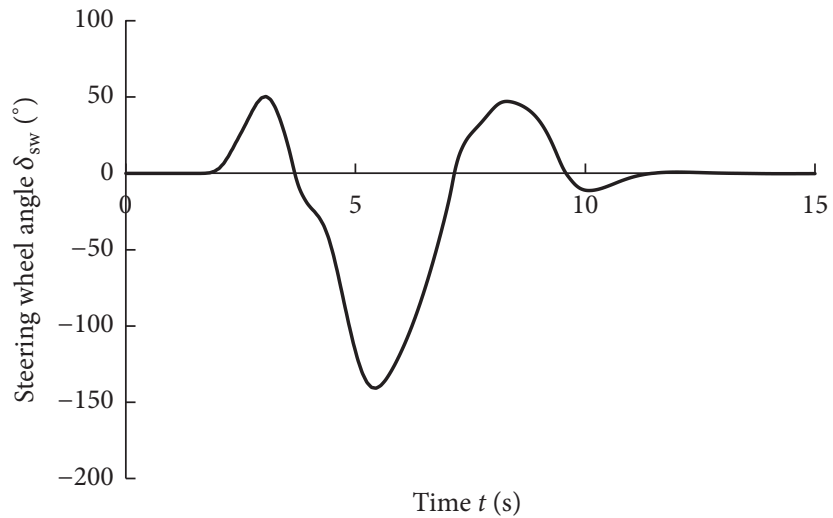

(c) Steering wheel angle

FIGURE 6: Vehicle state $(\mu=0.2)$.

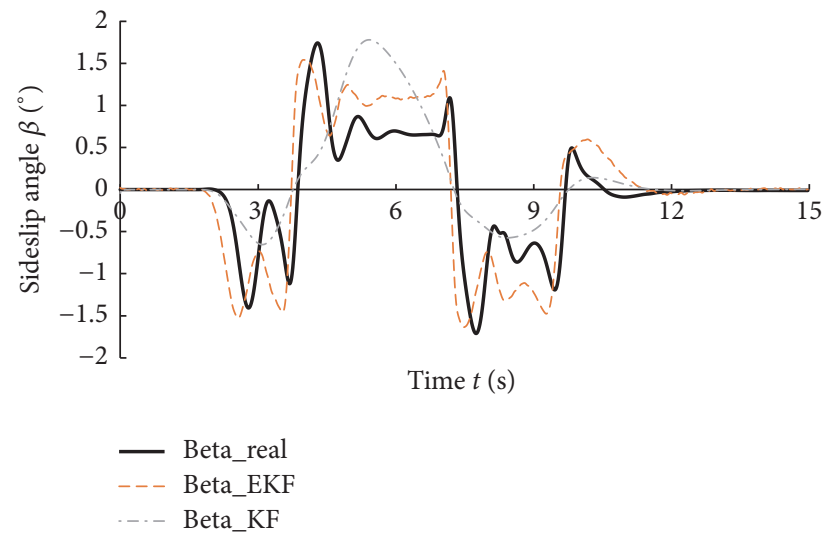

FIGURE 7: The contrast of sideslip angle simulation value and estimated value $(\mu=0.2)$. 


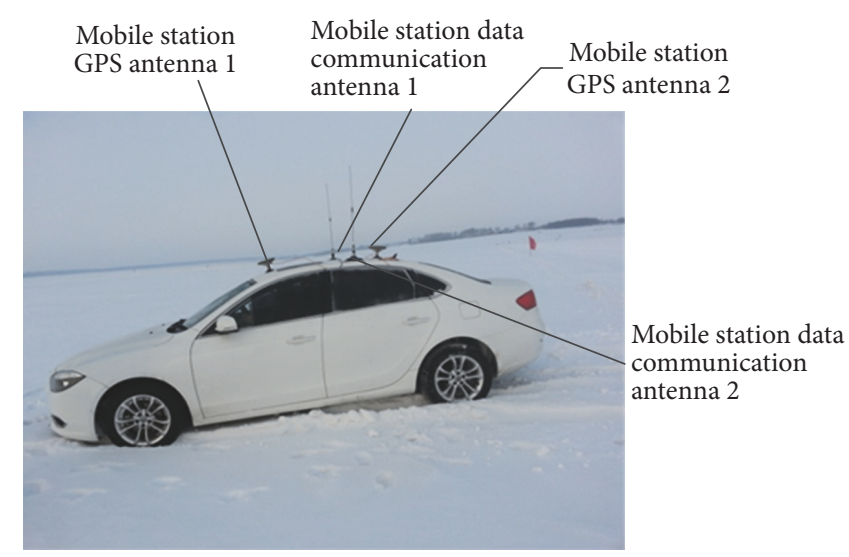

FIGURE 8: Arrangement of sideslip angle measurement system based on GPS.

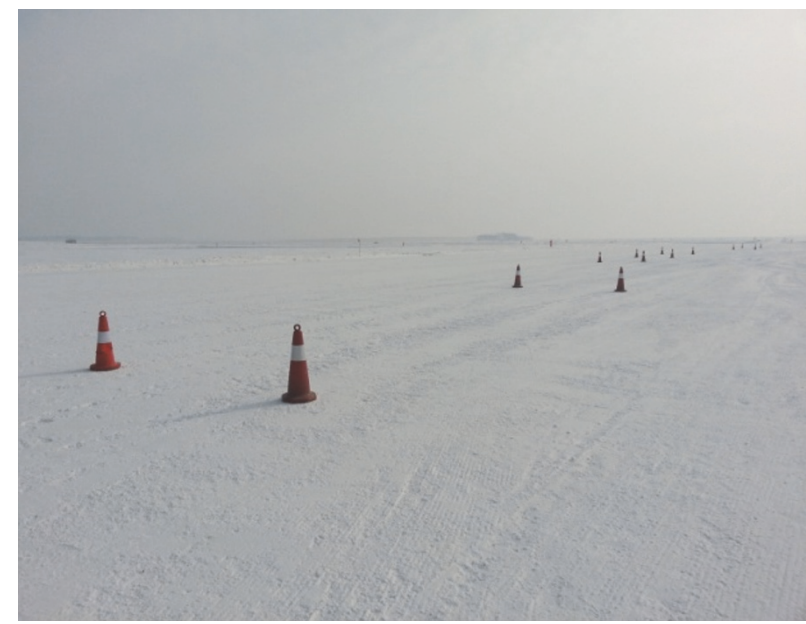

FIGURE 9: Double lane change test site.

\section{Sideslip Angle Estimation of Vehicle Test Method}

In the simulation test, the extended Kalman filtering algorithm has been verified. This section uses the real vehicle test data to estimate the sideslip angle and real vehicle test data to estimate the sideslip angle. Usually, when the vehicle goes into the nonlinear region, the sideslip angle estimation will have a large deviation from the actual value. In order to enter the nonlinear state, we take the double lane change test in vehicle limit test site with low adhesion road in Heihe City. It is easy to get the vehicle state data of nonlinear region.

Figure 8 shows the arrangement of sideslip angle measurement system, Figure 9 shows the snow road with $3 \mathrm{~cm}$ thick snow of the double lane test site. The adhesion coefficient of the actual ice and snow road will be changed to $0.3-0.35$ because of the difference of compaction degree, water content of snow, and smoothness of snow surface. The vehicle mounted gyroscope is used for real-time acquisition of vehicle yaw rate, lateral acceleration, vehicle speed, and steering wheel angle signal from the vehicle CAN communication.
According to ISO 3888-1: 1999 to implement the double lane change test, pass the double lane change path with the speed of $67 \mathrm{~km} / \mathrm{h}( \pm 5 \mathrm{~km} / \mathrm{h})$. At the same time, record the data in computer through Kvaser and write the time history curve of each measurement variable such as the steering wheel angle, yaw velocity, lateral acceleration, longitudinal velocity, the sideslip angle, and the steering rod force information.

Figure 10 shows the steering wheel angle, yaw velocity, longitudinal velocity, lateral acceleration, and yaw angle response curves. From Figure $10(\mathrm{~d})$, we can see that, in the first 10 seconds, the speed is $67 \mathrm{~km} / \mathrm{h}$ and in double lane change operation speed has decreased. In 20 seconds, the test speed was $60 \mathrm{~km} / \mathrm{h}$. From Figure 10(b), we can see that, in 14,5 seconds, the lateral acceleration has reached $0.33 \mathrm{~g}$ and the adhesion coefficient of snow and ice road surface is about 0.35. It is almost near the limit state of critical instability. Figure 10(e) shows the sideslip angle curve measurement. It has reached $3.22^{\circ}$ in $14.75 \mathrm{~s}$ and over $4^{\circ}$ in $16 \mathrm{~s}$. From Figures 10(b) and 10(e), we can see that, taking double lane test on snow and ice road with the speed of $67 \mathrm{~km} / \mathrm{h}$, the vehicle is near critical unstable state. 


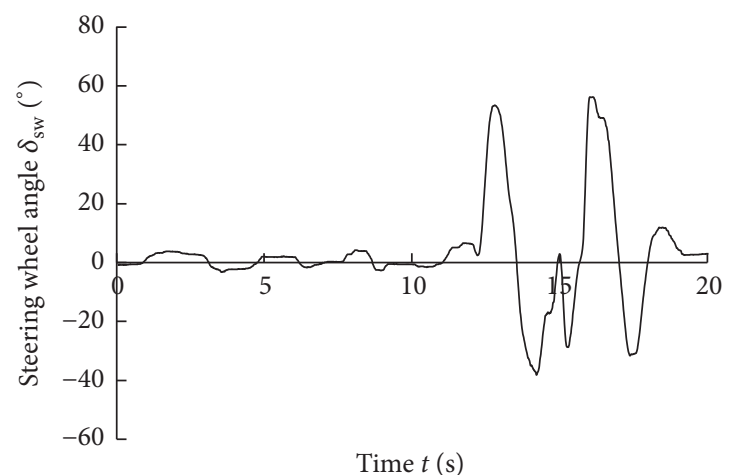

(a) Steering wheel angle

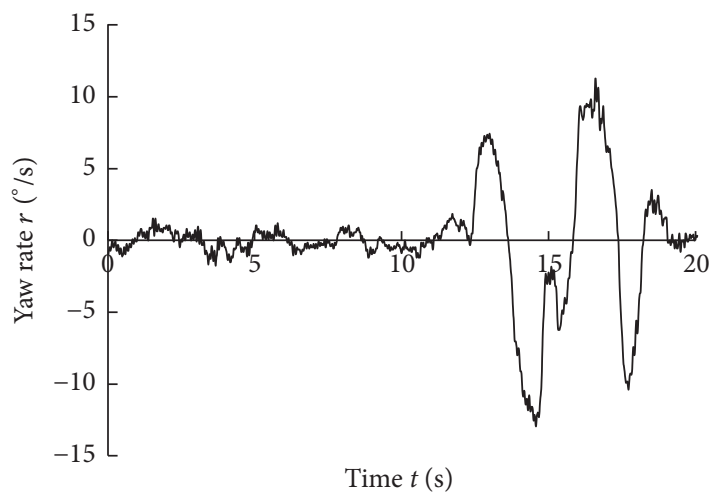

(c) Yaw rate

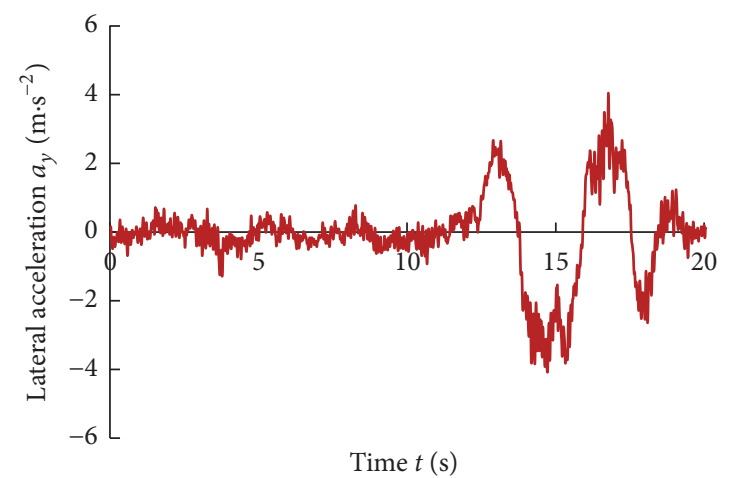

(b) Lateral acceleration

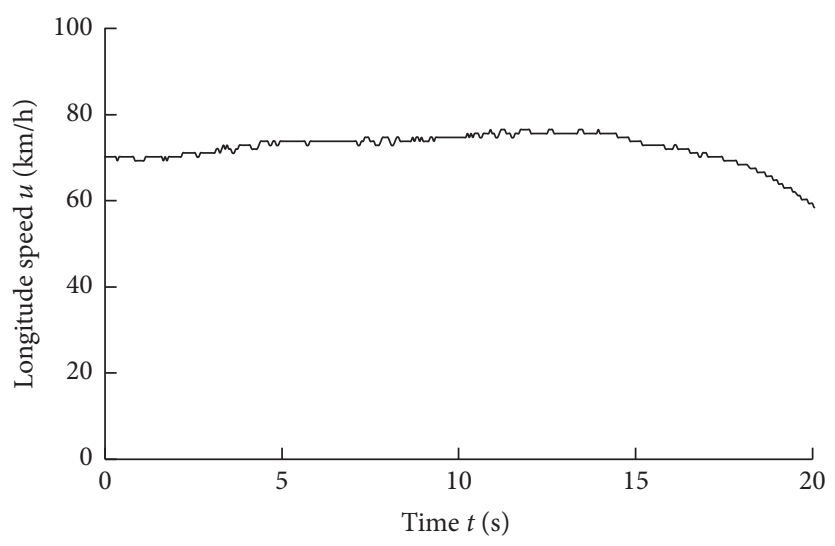

(d) Longitudinal speed

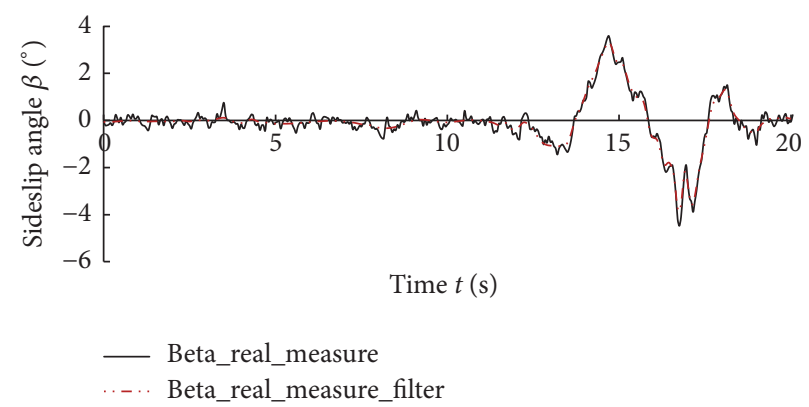

(e) Sideslip angle measurement and filtering value

FIGURE 10: Vehicle state parameters under line condition.

Figure 11 shows that, from the contrast among the extended Kalman filtering estimation, Kalman filtering estimation, and the measured value of the sideslip angle, we can see that when the vehicle is near the critical instability region, because Kalman filtering algorithm ignores the nonlinear tire, the estimation accuracy is lower than that of the extended Kalman filter algorithm.

\section{Conclusion}

This paper discusses Kalman filtering and extended Kalman filtering, the two kinds of algorithms of the sideslip angle estimation, and analyzes their characteristics. This paper presents the simulation of the two algorithms on the low adhesion road and carries out the low adhesion road double lane change test. The experimental results show that when the vehicle dynamics enter the nonlinear region, the accuracy of the sideslip angle estimation is higher than the accuracy of Kalman filtering algorithm. We will improve the algorithm to restrain the filtering divergence in the following study.

\section{Conflicts of Interest}

The authors declare that there are no conflicts of interest regarding the publication of this paper. 


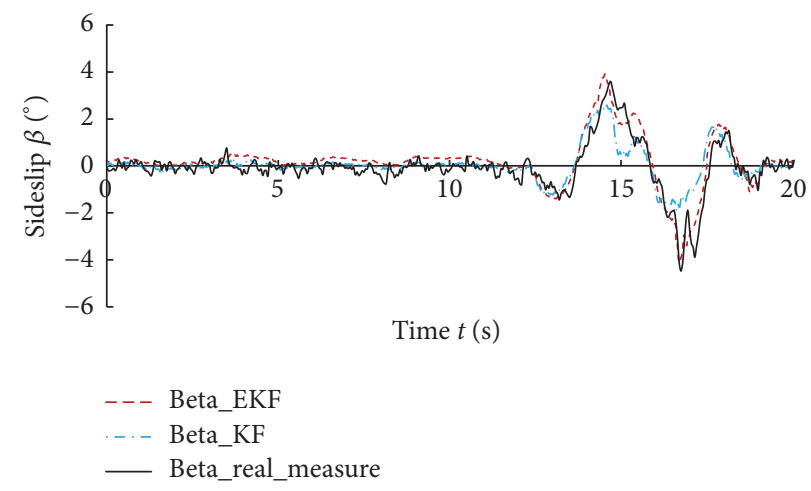

FIGURE 11: Sideslip angle identification values compared with the experimental value under line condition.

\section{Acknowledgments}

The authors acknowledge the support from Natural Science Foundation of Shandong Province (ZR2016EEQ06).

\section{References}

[1] M. Kang, L. Li, H. Li, J. Song, and Z. Han, "Coordinated vehicle traction control based on engine torque and brake pressure under complicated road conditions," Vehicle System Dynamics, vol. 50, no. 9, pp. 1473-1494, 2012.

[2] L. Li, J. Song, H.-Z. Li, D.-S. Shan, L. Kong, and C. C. Yang, "Comprehensive prediction method of road friction for vehicle dynamics control," Proceedings of the Institution of Mechanical Engineers, Part D: Journal of Automobile Engineering, vol. 223, no. 8, pp. 987-1002, 2009.

[3] G. Baffet, A. Charara, D. Lechner, and D. Thomas, "Experimental evaluation of observers for tire-road forces, sideslip angle and wheel cornering stiffness," Vehicle System Dynamics, vol. 46, no. 6, pp. 501-520, 2008.

[4] E. F. Camacho, Model Predictive Control, Springer, London, UK, 2007.

[5] L. Li, J. Song, H. Li, and X. Zhang, "A variable structure adaptive extended Kalman filter for vehicle slip angle estimation," International Journal of Vehicle Design, vol. 56, no. 1-4, pp. 161-185, 2011.

[6] C. Huang, L. Chen, C. Yun et al., "Fuzzy chaos control for vehicle lateral dynamics based on active suspension system," Chinese Journal of Mechanical Engineering, vol. 27, no. 2, pp. 304-318, 2014.

[7] H. Chen, C. Long, C.-C. Yuan, and H.-B. Jiang, "Non-linear modelling and control of semi-active suspensions with variable damping," Vehicle System Dynamics, vol. 51, no. 10, pp. 15681587, 2013.

[8] A. Y. Ungoren, H. Peng, and H. E. Tseng, "Experimental verification of lateral speed estimation methods," in Proceedings of the AVEC 6th International Symposium, Hiroshima, Japan, September 2002.

[9] M. C. Best, T. J. Gordon, and P. J. Dixon, “An extended adaptive Kalman filter for real-time state estimation of vehicle handling dynamics," Vehicle System Dynamics, vol. 34, no. 1, pp. 57-75, 2000.

[10] H. B. Pacejka, Type and Vehicle Dynamics, Butterworth-Heinemann, Oxford, UK, 2002.
[11] F. Chenli, E. Sabbioni, M. Pesce, and S. Melzi, "A methodology for vehicle sideslip angle identification: comparison with experimental data," Vehicle System Dynamics, vol. 45, no. 6, pp. 549$563,2007$.

[12] J. Stephant, A. Charara, and D. Meizel, "Virtual sensor: application to vehicle sideslip angle and transversal forces," IEEE Transactions on Industrial Electronics, vol. 51, no. 2, pp. 278-289, 2004.

[13] Y. Fukada, "Slip-angle estimation for vehicle stability control," Vehicle System Dynamics, vol. 32, no. 4, pp. 375-388, 1999.

[14] X. Y. Yang, Q. Qin, and Y. Y. Cui, “4WID in-wheel motor EV driving state estimation based on UKF," Applied Mechanics and Materials, no. 246, pp. 256-274, 2013. 


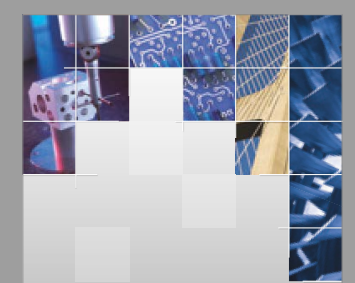

\section{Enfincering}
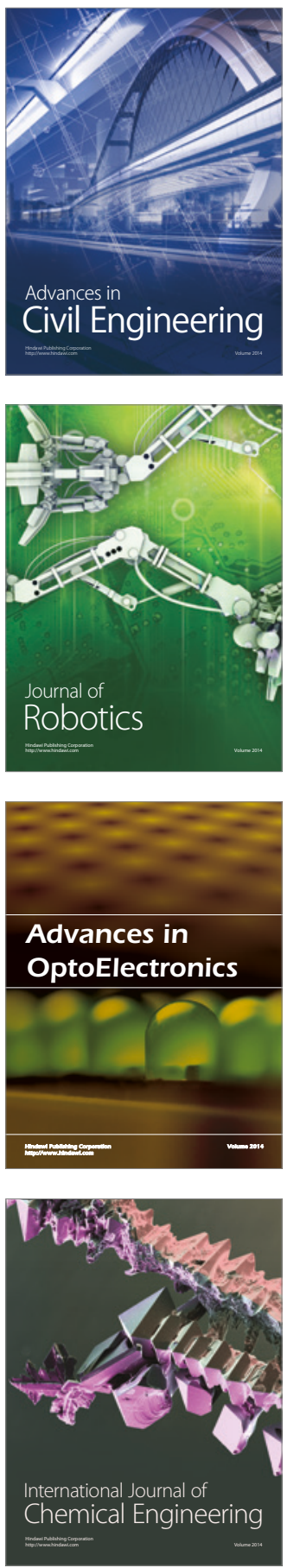

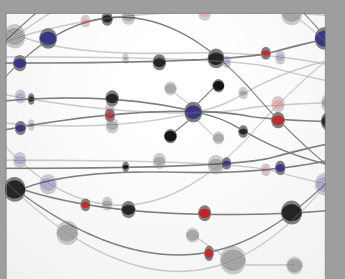

The Scientific World Journal

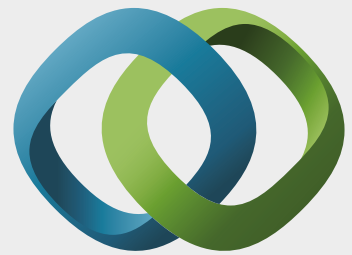

\section{Hindawi}

Submit your manuscripts at

https://www.hindawi.com
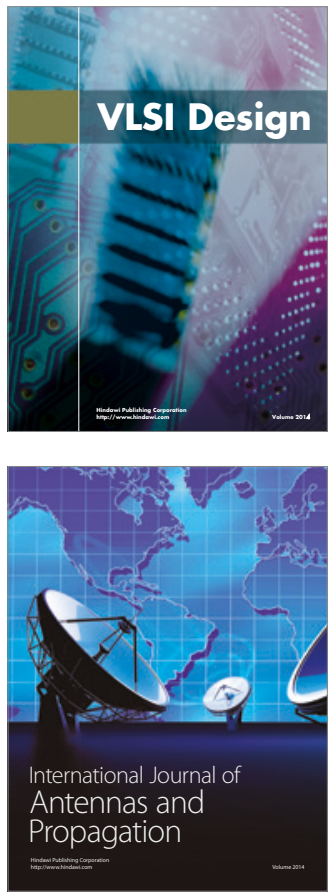

\section{Rotating}

Machinery
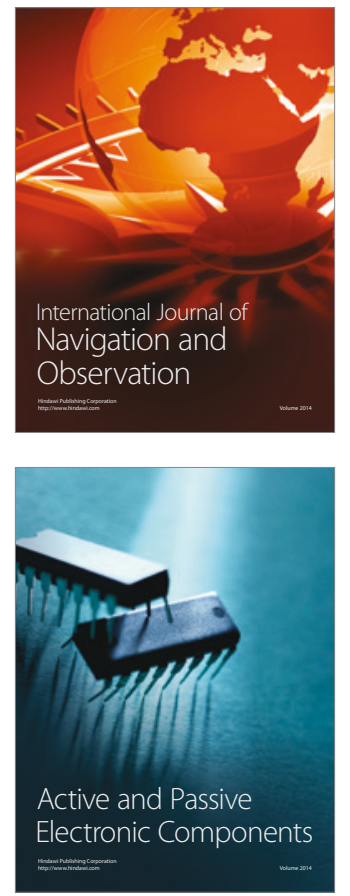
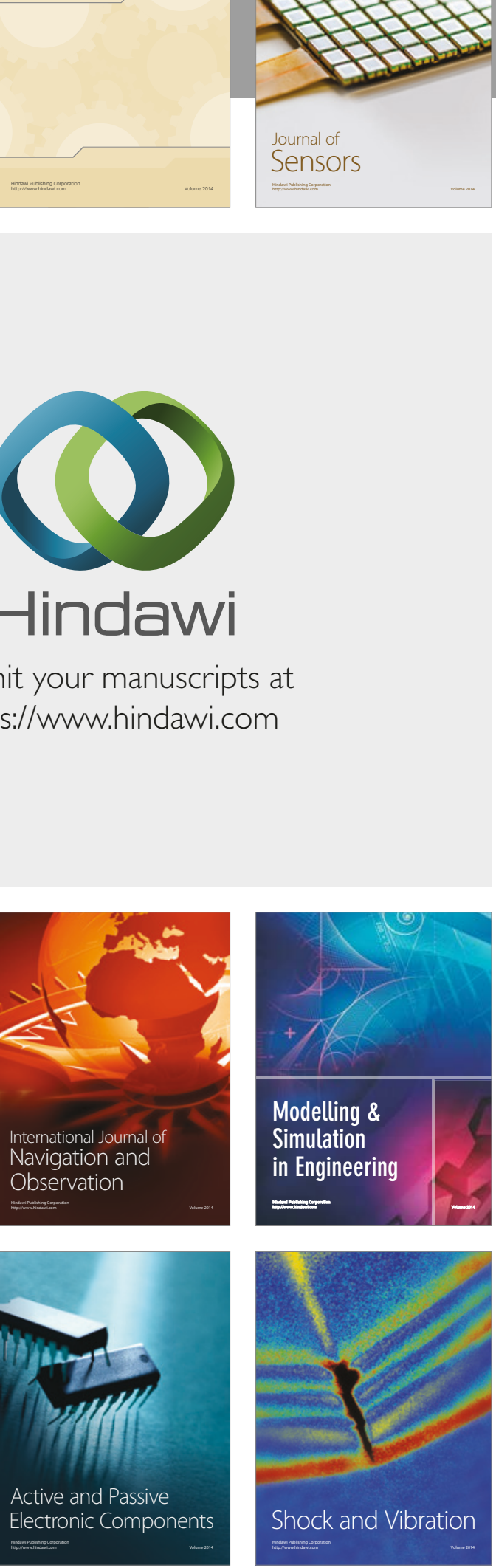
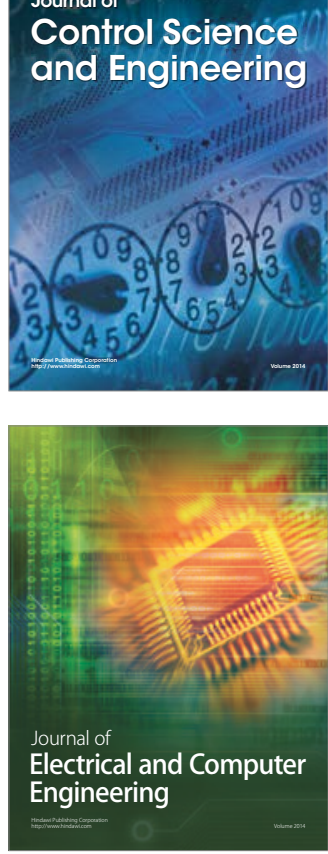

Distributed

Journal of

Control Science

and Engineering
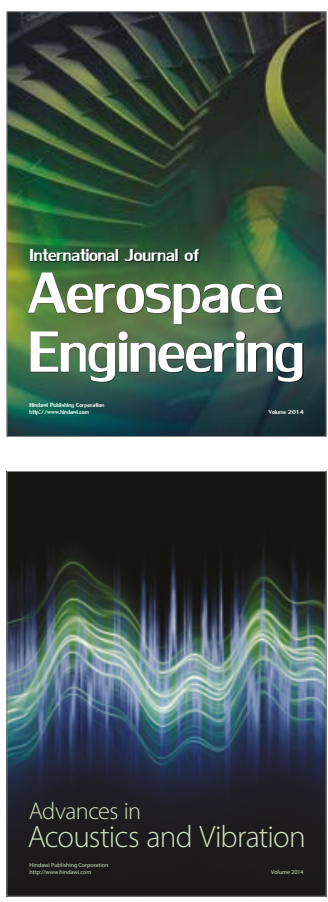

Sensor Networks 